\title{
ERRATUM
}

doi:10.1038/nature08493

\section{Stable single-unit-cell nanosheets of zeolite MFI as active and long-lived catalysts}

Minkee Choi, Kyungsu Na, Jeongnam Kim, Yasuhiro Sakamoto, Osamu Terasaki \& Ryong Ryoo

Nature 461, 246-249 (2009)

In this Letter, the affiliations for Osamu Terasaki were incorrect. This author is associated with affiliations 4 and 5 from this Letter, the Graduate School of EEWS (WCU), KAIST, and Structural Chemistry, Arrhenius Laboratory, Stockholm University, and not with Nanoscience and Nanotechnology Research Center, Osaka Prefecture University. 